\title{
THEORY AND PRACTICE OF VIBRATION ANALYSIS IN ELECTRONIC PACKAGES
}

\author{
Igor Kovtun ${ }^{1, *}$, Juliy Boiko², Svitlana Petrashchuk ${ }^{1}$, and Tomasz Kałaczyński ${ }^{3}$ \\ ${ }^{1}$ Technologies and Design Faculty in Khmelnytsky National University, Department of Art and \\ Technical Graphics, 29016, 11 Institutska street, Khmelnytsky, Ukraine \\ ${ }^{2}$ Programming and Computer, Telecommunication Systems Faculty in Khmelnytsky National \\ University, Department of Telecommunication and Radio Engineering, 29016, 11 Institutska street, \\ Khmelnytsky, Ukraine \\ ${ }^{3}$ Faculty of Mechanical Engineering UTP in Bydgoszcz, Department of Vehicle Engineering, Al. \\ Prof. S.Kaliskiego 7, 85-796 Bydgoszcz, Poland
}

\begin{abstract}
The paper represents theoretical and experimental vibration analysis of electronic packages. The research objectives are bearing parts, such as printed circuit boards, walls of the cases and electronic components, in enclosed electronic packages subjected to vibration during their operation. The theoretical model of the oscillation system representing electronic package installed on the support subjected to vibration is described. Vibration transmission from the shaker to the tested electronic package is explained as the complex problem to solve. The experimental measurement verified significant difference in amplitudes of vibrations transmitted inside the package and incident to. The elements of plate bending theory is used for main boards, case walls and other planar rectangular structures considered as equivalent homogeneous plates, in order to estimate their natural frequencies and forms of free cross oscillations and hence to identify their possible resonances with purpose to avoid them in operation. The significant attention was paid to the experimental research of dynamic characteristics, representing results in form of Chladni figures and amplitude frequency responses.
\end{abstract}

\section{Introduction}

Practice of vibration tests and also usage of electronic packages installed on the supports sustaining vibration during their operation testified of damages in some components or failures of electronic packages although the vibrations remained under acceptable limits [1]. Vibration measured at locations where such damages occurred indicated that at some excitation frequencies the damaged components and parts sustained vibration amplitude, velocity or acceleration (vibration overloads) 10 times higher than correspondent parameters simultaneously measured on the shaker table or on the support of electronic package that receives and transmits vibration while operation.

In case when printed circuit boards (PCBs) and other bearing parts of electronic packages sustain resonance oscillations the dynamic loads multiply and may cause breakages of components and units or malfunctions because of deviations of their electronic parameters [2].

Therefore, special attention is paid to finding natural oscillation frequencies of bearing parts in electronics by using calculative and experimental methods in order to identify their possible resonances and hence to avoid them and correspondent dangerous operational modes.

\footnotetext{
${ }^{\star}$ Corresponding author: dr.igorkovtun@gmail.com
} 
Complexity of this problem arises due to the lack of input data needed for calculations. As the matter of fact electronic packages represent complex structures comprising many components whose vibration characteristics such as mass and stiffness are different [2] and the knowledge of their real values is insufficient. Moreover, characteristics of components mounted on PCB and in this way connected are no longer individual and may vary as being influenced by other nearly situated components during vibration.

In calculations the main boards, case walls and other planar rectangular structures are normally considered as equivalent homogeneous plates [3] whose theory is quite complicated, long, full of simplifications and assumptions, and incomplete.

Therefore, the current study is focused on using elements of these theories and basic formulas in order to calculate and estimate natural frequencies of free cross oscillations of the rectangular plates and assess their efficiency and applicability to the planar construction elements of the electronic packages in particular to PCBs. The significant attention was paid to the experimental research conducted by vibration tests, representing results in form of Chladni figures and amplitude frequency response, which allowed assessing vibration for the real structures subjected to the real operational conditions.

\section{Theoretical model of oscillation system}

The dynamic characteristics of any object including electronic packages are normally measured while vibration tests performed on the shakers. The shaker with the tested object installed on it and the clamps fastening the object to the shaker are the elements of one oscillation system. In the common case such oscillation system can be represented by the simplified mass-spring-damper model shown in fig. 1. The tested object and the clamps are assumed as the total mass $m$.

This installation is not considered as absolutely rigid therefore the model (fig. 1) is represented as the total mass $m$ supported by the shaker by the spring with stiffness $k$ and the damper with damping coefficient $c$.

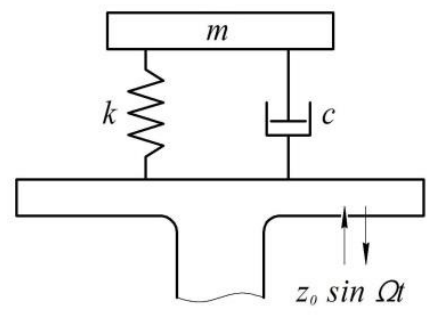

Fig. 1. Oscillation model.

The movable shaker table (shaker) generates harmonic oscillations described by the function $z_{0} \sin \Omega t$, where $z_{0}$ - amplitude, $\Omega$ - angular frequency, $t$ - time of oscillations.

Downward movement of the shaker by the value $z_{0} \sin \Omega t$ causes spring extension that is expressed by formula $z-z_{0} \sin \Omega t$, where $z$ stands for mass vertical downward displacement. The elastic force of the spring is expressed as $-k\left(z-z_{0} \sin \Omega t\right)$ and the damping force proportional to velocity is $-c\left(z^{\prime}-z_{0} \Omega \cos \Omega t\right)$.

On the base of second Newton's law the following oscillation equation of the system is obtained:

$$
\begin{gathered}
m z^{\prime \prime}=-k\left(z-z_{0} \sin \Omega t\right)-c\left(z^{\prime}-z_{0} \Omega \cos \Omega t\right), \\
m z^{\prime \prime}+k\left(z-z_{0} \sin \Omega t\right)+c\left(z^{\prime}-z_{0} \Omega \cos \Omega t\right)=0 \\
\left.m z^{\prime \prime}+c z^{\prime}+k z=k z_{0} \sin \Omega t+c z_{0} \Omega \cos \Omega t\right)
\end{gathered}
$$


Mass $m$ movement with respect to vibrating shaker is found by spring extension derivatives taken with assumption that mass $m$ displaced at the initial moment together with the spring by $z_{1}=z-z_{0} \sin \Omega t$ then $z=z_{1}+z_{0} \sin \Omega t$.

Derivatives are:

$$
z^{\prime}=z_{1}^{\prime}+z_{0} \Omega \cos \Omega t, z^{\prime \prime}=z_{1}^{\prime \prime}-z_{0} \Omega^{2} \sin \Omega t .
$$

Substituting these expressions into equation 1 gives the expression:

The final simplified expression is:

$$
\begin{gathered}
m z_{1}^{\prime \prime}-m z_{0} \Omega^{2} \sin \Omega t+c z_{1}^{\prime}+c z_{0} \Omega \cos \Omega+k z_{1}+k z_{0} \sin \Omega t= \\
=k z_{0} \sin \Omega t+c z_{0} \Omega \cos \Omega t
\end{gathered}
$$

$$
m z_{1}^{\prime \prime}+c z_{1}^{\prime}+k z_{1}=m z_{0} \Omega^{2} \sin \Omega t
$$

where $m z_{1}^{\prime \prime}-$ mass inertia; $c z_{1}^{\prime}-$ damping force; $k z_{1}-$ spring elastic force; $m z_{0} \Omega^{2}-$ inertial exciting force.

Such excitation of oscillation is called inertial [4].

Thus, the statements is made that the mass $m$ (which includes both tested object and clamping appliance) moves relative to vibrating base (shaker table) in the same way as the mass $m$ moves on motionless base (shaker), when mass is exposed to harmonic excitation force by amplitude $m z_{0} \Omega^{2}$. Forced oscillations of such single-mass system have been well studied and are not in objective for the present research.

Noteworthy is that amplitude and phase of such oscillations depend on proximity of excitation force frequency $\Omega$ to cycling frequency $\omega_{c}(5)$ of natural oscillations for mass $m$.

$$
\omega_{c}=\sqrt{\frac{k}{m}} .
$$

The formula for oscillation of the system with mass $m$, stiffness $k$, and damping $c=0$ is:

$$
z=\frac{z_{0} \sin \Omega t}{1-\left(\frac{\Omega}{\omega_{c}}\right)^{2}}
$$

Thus, the issue is made that mass oscillation becomes close to shaker oscillation only when $\omega_{c}$ is considerably higher than $\Omega$.

As $\Omega$ approaches to $\omega_{c}$, the mass oscillation grows and goes out of phase.

When $\Omega=\omega_{c}$ the system undergoes resonance so that mass oscillation tends to maximum $(\infty)$ and phase difference tends to $90^{\circ}$.

After passing the resonance and further more after, mass oscillation decreases approaching to zero and phase delay increases so the phase difference ultimately reaches $180^{\circ}$. Thus, mass oscillations (in the tested object) will be directed contra-phase to the shaker oscillation and will likely quench them. Actually, since $\Omega=10 \omega$ the oscillations of the shaker will not be transmitted to the mass $m$.

Theory of two-mass systems assumes possibility to quench oscillations of one mass by the contra-phase oscillations of another one connected by the spring. This is the foundation of oscillation dynamic quench principle. However, this issue is not for consideration in the present paper. The important issue, which is theoretically proven, is so that if mass installation on the shaker is not absolutely rigid then shaker oscillation does not have to coincide with that of the mass.

Incidentally, the shaker oscillations are desirable to be transmitted from the shaker to the tested object without distortion at 1:1 scale for all excitation frequencies. But the reality appears to be even more complicated.

\section{Vibration tests of electronic blocks}

The vibration tests were performed for electronic blocks representing electronic packages in the enclosure case. The type of blocks chosen for the test had damages detected during typical 
production tests. Vibration graphing was applied during the test. Five vibration sensors were installed in places of potential damages on the case surface (fig. 2).

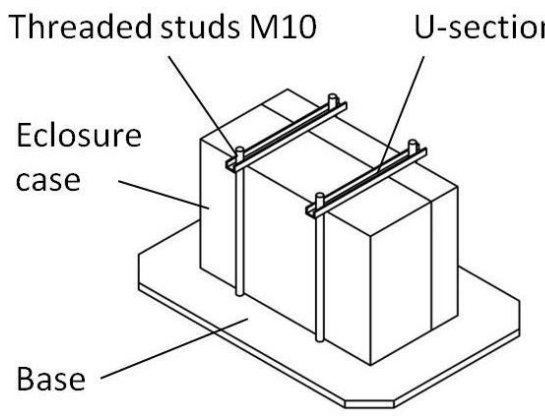

a)

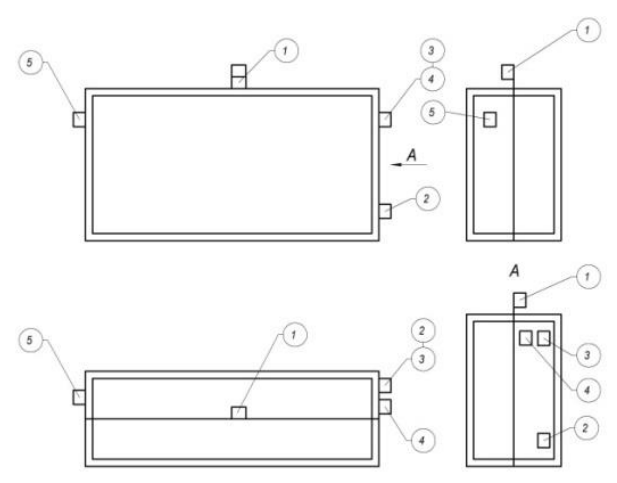

b)

Fig. 2. Vibration test installation: a) clamping appliance; b) vibration sensors installation.

The vibration sensor 6 was set on the shaker platform (not shown in the fig. 2). Then in accordance to production testing technology the tested block was fastened on the base by four threaded studs M10 and two U-sections; the base was tightened to the shaker platform by two bolts M10. It should be mentioned that production technology did not provide for the bolts fastening control.

The experiment was conducted in the following order. In accordance to the technical specifications the blocks were subjected to vibration tests by the fixed frequency method. The shaker was set to the specific (frequency and amplitude) oscillation mode.

Vibration sensors were connected to vibration measuring instruments. The given oscillation frequency was controlled by the sound generator.

To identify resonance of the individual parts or the whole block in given frequency range of vibration the sweep frequency method was applied.

The measurement results were obtained at these resonance frequencies at specified and constant amplitude of the shaker oscillation $(\mathrm{A}=0.1 \mathrm{~mm})$.

Analysis of collected data testified that oscillation amplitudes, recorded from vibration sensors on the tested block, 8-25 times exceeded the generated ones. For an instance, the real oscillation amplitude reported by vibration sensors reached from $1.35 \mathrm{~mm}$ (sensor 2) to 1.85 $\mathrm{mm}$ (sensor 1) and $2.7 \mathrm{~mm}$ (sensor 5) at oscillation frequency $80 \mathrm{~Hz}$ whilst the shaker amplitude was $0.1 \mathrm{~mm}$. During the tests conducted at frequencies lower than $70 \mathrm{~Hz}$ this difference was negligible.

Another bearing block was subjected to vibration test. To identify overloads in PCBs inside the block two piezosensors ABC 032 were installed. The generated overloads were controlled by another two piezosensors set in the places of block attachment onto the shaker table.

The experiment was conducted as follows. The tested block with sensors attached was installed on the platform of the shaker in two positions: horizontally when vibration was directed along the PCBs maximal stiffness; and then at the side position when direction of vibration coincided with direction of PCBs minimal stiffness (excitation force was directed perpendicularly to the PCB plane).

Then the block was exposed to vibration acceleration of about 4-14 $\mathrm{g}$ read from vibration sensors 1 and 2, which were set in places of block attachment to the shaker, and vibration overloads of PCB were read from sensors 3 and 4 . This procedure was conducted in both horizontal and side positions of the tested block. 
The vibration overloads of PCB measured during the tests at the resonance frequencies with vibration acceleration driven to the places of block attachment demonstrate that vibration sensors set in different places of the shaker table measured different vibrations, what testifies that vibration unevenly distributed over the shaker table, and also that PCB vibration at resonance excitation may differ from vibration driven to places of block attachment by dozens of times, what confirms theoretical results obtained before.

\section{Elements of plate vibration theory}

Printed circuit boards, case walls and other planar rectangular structures used in electronic packages are represented as homogeneous rectangular plates to perform their vibration analysis and calculations. The plate is assumed an object of geometry, in which height $\mathrm{H}$ is at least 5 times less than its length and width.

The coordinate origin is oriented in the plate so that axes $\mathrm{X}$ and $\mathrm{Y}$ are constraint to its middle plane to which axis $\mathrm{Z}$ is perpendicular. The cross uniform inertial load bends the plate and its mid-plane becomes the middle elastic surface, which divides the plate into stretched and contracted areas. The same assertion refers to neutral fibers and neutral section axis in beams theory [4]. As is the case with beams vertical displacements of points in mid-surface are called plate deflections $z$, which are assumed small in comparison with plate thickness $H$. As well as for the case of beams the assumption can be made within second order accuracy that when the plate is deflected then the fibers of its mid-surface do not change their length, what means that analogically to neutral axis and neutral fibers the mid-surface is the neutral one and all its points receive displacement along axis $\mathrm{Z}$ while deformation.

Analogically to the planar section hypothesis in beam bending theory [4] the kinematic hypothesis or straight normal hypothesis is used in plate theory, in accordance to which the planes, having been normal to middle plane of the plate before deformation, remain normal thereafter. The deformation image of the plate reminds that of the beam with an exception that it deforms in two planes $\mathrm{XZ}$ and $\mathrm{YZ}$ simultaneously.

Points in the mid-surface are assumed with the second order accuracy to receive only vertical displacements, which are deflections $z$, and points vertically remote from mid-surface sustain more than just vertical displacements but also horizontal ones, which are derived from displacements along axes $\mathrm{X}$ and $\mathrm{Y}$.

In accordance to straight normal hypothesis each normal to mid-surface as being deformed displaces as absolutely rigid piece equal to plate thickness $H$, so all points of the deformed plate can be found by drawing perpendiculars of length $H / 2$ from correspondent mid-surface point. Thus, finding mid-surface equation $z=z(x, y, t)$ grants finding positions of all points in the plate and hereby using Hook's law finding stress and solving strength problems by using strength theories [4].

The general expression for the plate deflection, which is function $z(x, y, t)$ obtained by using mentioned theories is represented by the following formula:

$$
\frac{\partial^{4} z}{\partial x^{4}}+2 \frac{\partial^{4} z}{\partial x^{2} \partial y^{2}}+\frac{\partial^{4} z}{\partial y^{4}}+\frac{\rho H}{g D} \frac{\partial^{2} z}{\partial t^{2}}=\frac{q}{D}
$$

where $\rho$ - plate material density $\mathrm{kg} / \mathrm{cm}^{3} ; g$ - standard gravity, $\mathrm{m} / \mathrm{s}^{2} ; D$ - plate cylindrical stiffness, $\mathrm{kgf} \cdot \mathrm{cm} ; q$ - intensity of load distributed over the plate surface, $\mathrm{kg} / \mathrm{cm}^{2} ; t-$ time, $\mathrm{s}$.

Cylindrical stiffness $D$ is expressed as:

$$
D=\frac{E H^{2}}{12\left(1-\mu^{2}\right)}
$$

where $E$ - elastic modulus of the plate material, $\mathrm{N} / \mathrm{m}^{2} ; \mu$ - Poisson's ratio.

If necessary, the presentation of expression (1) can be shortened by implementing harmonic operator [4] named as Laplace operator:

$$
\Delta=\frac{\partial^{2}}{\partial x^{2}}+\frac{\partial^{2}}{\partial y^{2}}
$$

and biharmonic operator: 


$$
\Delta \Delta=\left(\frac{\partial^{2}}{\partial x^{2}}+\frac{\partial^{2}}{\partial y^{2}}\right)\left(\frac{\partial^{2}}{\partial x^{2}}+\frac{\partial^{2}}{\partial y^{2}}\right)=\frac{\partial^{4}}{\partial x^{4}}+2 \frac{\partial^{4}}{\partial x^{2} \partial y^{2}}+\frac{\partial^{4}}{\partial y^{4}} .
$$

Then the shortened equation for the plate becomes written as:

$$
\Delta \Delta(z)+\frac{\gamma H}{g D} \frac{\partial^{2} z}{\partial t^{2}}=\frac{q}{D} .
$$

The case can be considered more particularly. When $q$ is function of time $t$, then equation (7) describes forced cross oscillation of plate driven by time variant distributed load $q$; when $q$ is constant and time invariant, then plate sustains free or natural cross oscillation; when $q$ $=0$, then the following equation is obtained:

$$
\Delta \Delta(z)+\frac{\gamma H}{g D} \frac{\partial^{2} z}{\partial t^{2}}=0
$$

which describes free oscillations of unloaded plate.

A problem that describes free oscillation assumes the inertial force as the load:

$$
m \cdot a=\frac{\gamma H}{g} \frac{\partial^{2} z}{\partial t^{2}}=m \frac{\partial^{2} z}{\partial t^{2}},
$$

where $a$-acceleration, $\mathrm{m} / \mathrm{s}^{2} ; \frac{\gamma H}{g}=m$ - plate mass, $\mathrm{kg}$.

Plugging this expression into (6) gives:

$$
D\left(\frac{\partial^{4} z}{\partial x^{4}}+2 \frac{\partial^{4} z}{\partial x^{2} \partial y^{2}}+\frac{\partial^{4} z}{\partial y^{4}}\right)+m \frac{\partial^{2} z}{\partial t^{2}}=0 .
$$

\section{Natural frequency and form of oscillation}

For the case when all edges of the plate freely rest on supports solution of equation (14) is:

$$
z(x, y, t)=w(x, y) e^{j \omega_{0} t}
$$

The amplitude function $w(x, y)$, which is called natural oscillation form of the plate is defined as:

$$
w(x, y)=A_{u, f} \sin (u \pi x / a) \cdot \sin (f \pi y / b),
$$

where $a, b$ - length and width of the plate correspondently; $u, f$-number of sinusoid halfwaves in direction of axes $\mathrm{X}$ and $\mathrm{Y}$ correspondently.

Dependence (15) is demonstrated in fig. 3. Any line parallel to axis $X$ converts into sinusoid, which consists of $u$ half-waves in interval $(0, a)$, and the line parallel to axis $\mathrm{Y}$ has $f$ half-waves. As shown in fig. $3 u=2, f=3$.

Grid lines drawn from the nodes of half-waves divide plate into six sections, signs in which indicate direction of their deflection in specified moment of time: positive sign indicates upward deflection; negative sign - downward deflection, therewith signs shown in the left indicate deflection in ZY plane and those in the right - in ZX plane.

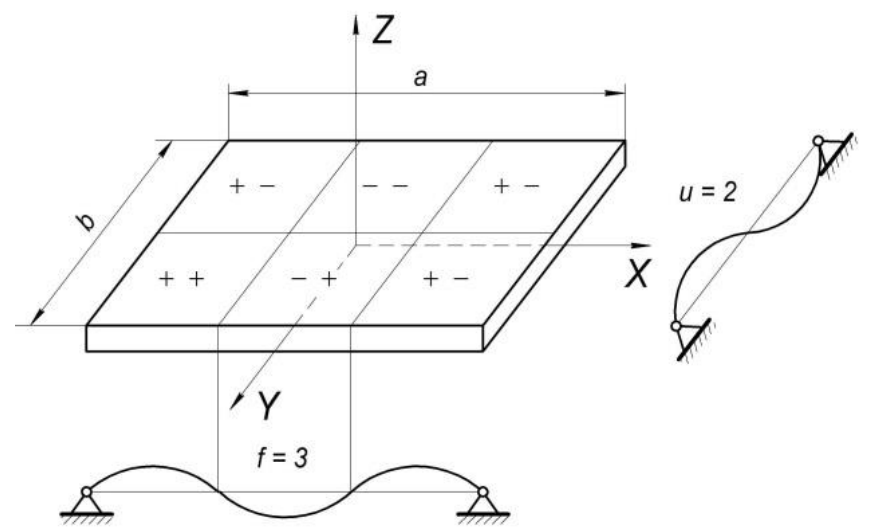

Fig. 3. Plate deformation and oscillation forms.

This solution complies with boundary conditions in outer edges: 
- $\quad z=0$ and $\frac{\partial^{2} z}{\partial x^{2}}+\mu \frac{\partial^{2} z}{\partial y^{2}}=0$ when $x=0$ and $x=a$;

- $z=0$ and $\frac{\partial^{2} z}{\partial y^{2}}+\mu \frac{\partial^{2} z}{\partial x^{2}}=0$ when $y=0$ and $y=b$ correspondently,

which indicate deflections and bending moments equal to zero in the edges (that rest on supports) of the plate.

Substitution of solutions $z(x, y, t)(15)$ and (16) into (12) gives:

$$
\left(\frac{u \pi}{a}\right)^{4}+2\left(\frac{u \pi}{a}\right)^{2} \cdot\left(\frac{f \pi}{b}\right)^{2}+\left(\frac{f \pi}{b}\right)^{4}=\omega_{0} \frac{\rho H}{D} .
$$

Wherefrom natural frequency of oscillation is:

$$
\omega_{0}=\pi^{2}\left[(u / a)^{2}+(f / b)^{2}\right] \sqrt{D / \rho H} .
$$

The represented method for finding natural frequency of oscillation is also applicable for the case when rectangular plate is freely supported on two parallel edges, what is normally the major method for PCBs installation in multilevel cases.

The fact is that real natural frequencies often differ from values estimated by demonstrated formula (18) because of the whole range of factors, such as: PCB population with discrete components and their non-uniform distribution over its area; conductive tracks, pads and other features etched from copper, which change stiffness of the PCB substrate; complexity to estimate fastening force in PCB fixtures to the cases of electronic packages and others. Noteworthy is that oscillations of PCB substrate and installed components are mechanically connected and in resonance frequencies the near components may involve each other into the mutual oscillations and in this way change parameters of the whole oscillation system: mass, stiffness, damping etc. Hence the conjecture is made that natural frequency estimation is just approximate and the real values can only be obtained experimentally.

To verify this conjecture the natural frequencies were calculated by formulas (18), and then measured experimentally. The research was conducted for the batch of PCBs with fiberglass epoxy substrate populated with integrated circuits uniformly distributed over its surface. The PCBs parameters were: $H=1.22 \mathrm{~mm} ; a=200 \mathrm{~mm} ; a / b=1 ; Q_{c} / Q_{b}=1$. The natural frequency calculated by the formula (18) equaled to $75 \mathrm{~Hz}$. Meanwhile the average value of natural frequencies measured experimentally was $150 \mathrm{~Hz}$.

Therefore, the represented estimation method is only considered as rough and approximate but accurate evaluation of natural oscillation frequency in the whole system of PCB substrate with installed components requires application of more complex models or experimental methods of the research.

\section{Chladni figures and frequency response}

The forms of oscillations for PCBs or electronic components were also offered to define by application of Hook's and Chladni method [5]. The idea of Hook's and Chladni method was applied to research forms of oscillations for PCBs using designed experimental installation (fig. 4).

The surface of the tested PCBs was wetted by kerosene and poured by fine-disperse quartz sand. Varying excitation frequency of vibration driven to the tested plate its oscillation amplitude rises at the resonance. This effect testifies of excitation and natural frequencies coincidence, what results in resonance. Therewith sand is dropped from the oscillation antinodes down to places, where board is not vibrating, which are considered to be oscillation nodes. In the nodes sand remains still. Besides, in these places the highest stresses appear. This method was successfully applied to find forms and natural frequencies of oscillation for main boards in four different variants of boards installation and also for bearing case walls in three orthogonal planes.

In the electronic packages PCBs are installed as in horizontal so in vertical positions what was provide by experimental cross appliance shown in fig. 4. The appliance provides simultaneous testing of $12 \mathrm{PCBs}$ as the maximum. 


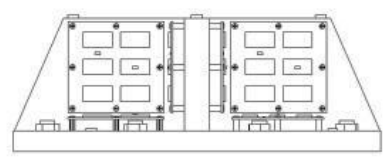

a)

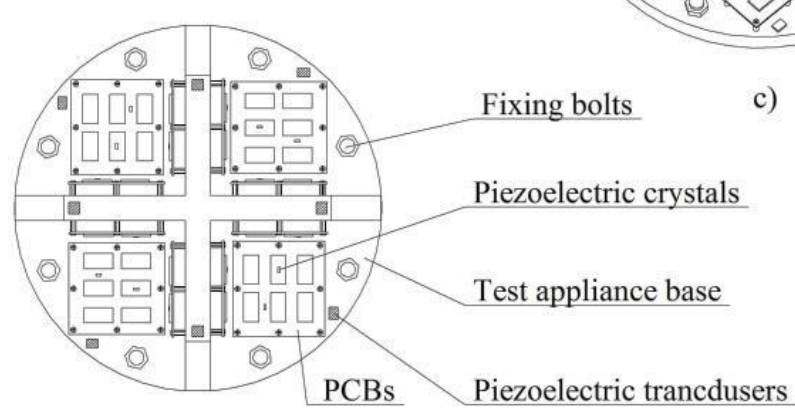

b)

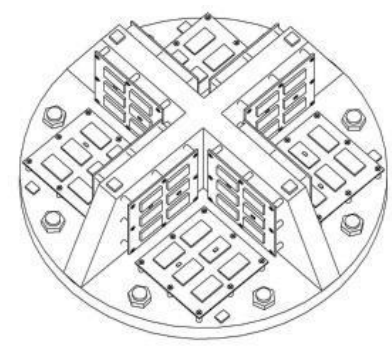

c)

Fig. 4. Experimental vibration testing cross appliance presentation: a) front; b) top; c) pictorial projections.

Tests were conducted for PCBs populated with three types of electronic modules, which differed by their elastic and inertial characteristics. Masses of tested electronic modules M1, M2, M3 were 60, 80, $100 \mathrm{~g}$ correspondently.

The natural frequencies, forms and amplitudes of oscillations of PCBs were searched in frequency diapason of $2000-5000 \mathrm{~Hz}$, which corresponds to operational conditions of electronic packages flying onboard. The test results are represented in form of Chladni figures (fig. 5, 6) and also by amplitude frequency responses, which were defined by averaged amplitude ratios $A_{m d l} / A_{b r d}$ (fig. 7), where $A_{m d l}$ and $A_{b r d}$ - oscillation amplitudes of electronic modules and the main boards correspondently. The amplitudes of oscillations in electronic modules were measured with consideration of irregular distribution of vibration acceleration over the shaker platform.

The Chladni figures demonstrate that researched objects have complex forms of oscillations, what testifies of complex strain and hence stress conditions for both boards and electronic modules [6].

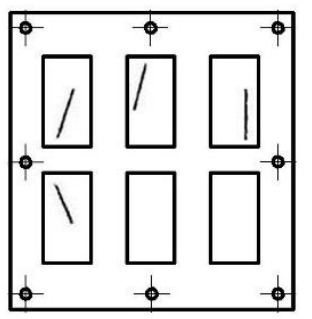

a)

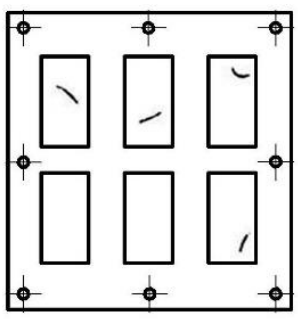

b)

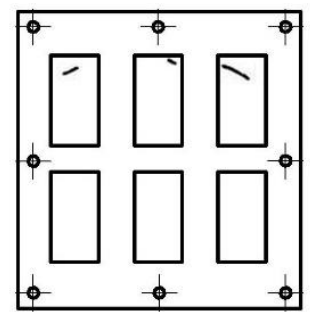

c)

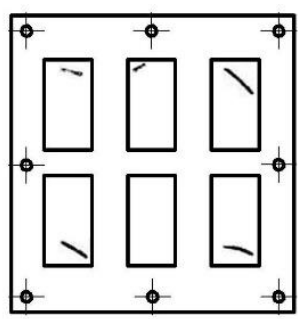

d) 


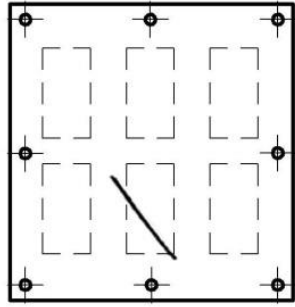

e)

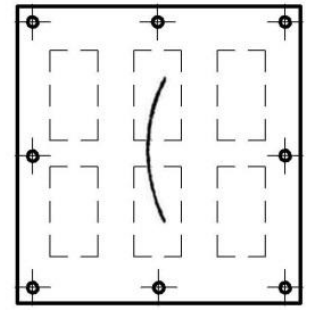

f)

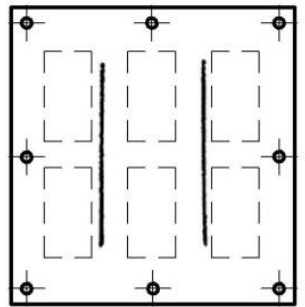

g)

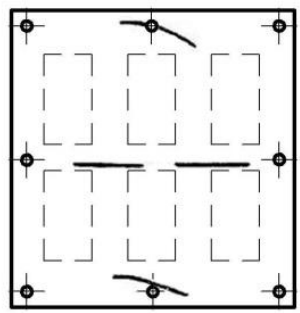

h)

Fig. 5. Chladni figures for PCBs installed in horizontal position and tested by vibration at their natural frequencies (Hz): a) 2580; b) 3373; c) 3501; d) 3666; e) 4880; f) 2061; g) 2227; h) 2799 .

Analysis of amplitude frequency responses (fig. 6) testifies of higher resonant response for boards installed in vertical position than in horizontal one. This fact is explained by the difference of elastic characteristics of tested objects which vary depending on whether vertical or horizontal installations. Besides, obtained results indicate of having dense spectrum of natural frequencies, which depends on design of electronic modules installed on the main boards.

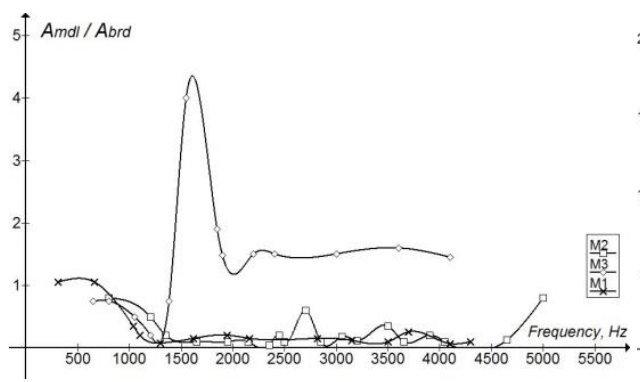

a)

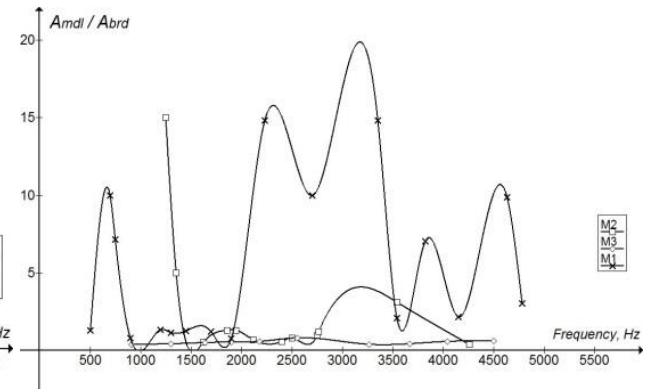

b)

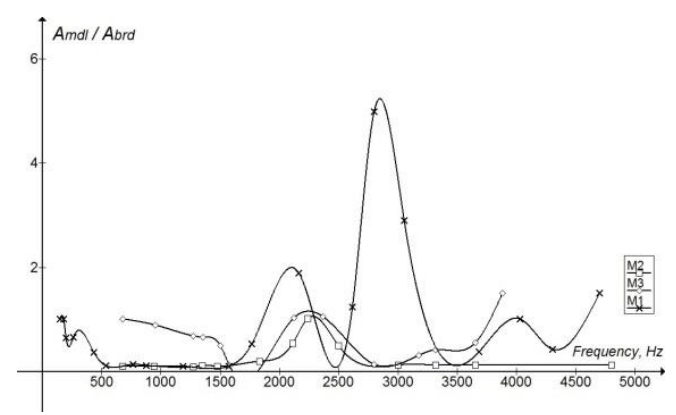

c)

Fig. 6. Amplitude frequency response for main boards installed in experimental vibration test cross appliance: a) vertically in X plane; b) vertically in Y plane; c) horizontally.

Thus, obtained experimental results testify that measuring dynamic characteristics of PCBs populated with electronic components or modules requires consideration of PCB installation position in the electronic package and electronic component distribution over the PCB area.

\section{Conclusions}

The theoretical model has been developed for oscillation system that represents electronic packages installed on supports subjected to vibration. The theoretically proven issue is made 
that vibration parameters of supports do not characterize vibration inside the electronic packages unless their connection is absolutely rigid.

Printed circuit boards, case walls and other planar rectangular structures used in electronic packages are represented as homogeneous rectangular plates in the offered plate bending model to perform their vibration analysis, however such estimation is approximate in comparison with the real values obtained experimentally.

Considering complexity, which becomes disadvantages of the traditional techniques for vibration tests performed by shakers, the experimental setup was created to define natural frequency and form of oscillation of PCBs and electronic components by using reverse and direct piezoelectric effects.

The Chladni figures, applied to vibration analysis of printed circuit boards and installed electronic components, demonstrated complex forms of oscillations, what testifies of complex strain and hence stress conditions for the research objects.

Analysis of amplitude frequency responses of printed circuit boards populated with electronic components testify that measuring their dynamic characteristics requires consideration of $\mathrm{PCB}$ installation position in the electronic package and electronic component distribution over the PCB area.

\section{References}

1. G.S. Aglietti, C. Schwingshackl. Analysis of Enclosures and Anti Vibration Devices for Electronic Equipment for Space Applications. School of Engineering Sciences, Aeronautics and Astronautics, University of Southampton, UK (2010)

2. T. Hamano, Y. Ueki, T. Nakasuji, K. Fujimoto. Destruction mechanisms resulting from vibration load in PCB-mounted electronics. ESPEC Technology Report No.17, 9th Symposium on Microjoining and assembly technology in electronics (2003)

3. R.M. Jones. Buckling of bars, plates and shells. Virginia, USA: Blacksburg, Bull Ridge Publishing (2006)

4. G.S. Pisarenko, V.A. Agarev. Strength of materials. (Kiev: Technika, 1967)

5. T. D. Rossing. Chladni's law for vibrating plates. American Journal of Physics, vol. 50, issue 3, pp. 271-274, 1982, doi.org/10.1119/1.12866

6. I. Kovtun, J. Boiko, S. Petrashchuk, G. Bauriene, K. Pilkauskas. Effects of the strain transmission from the main board to the installed electronic components. MECHANIKA, vol. 22(6), pp. 489-494, (2016), DOI: http://dx.doi.org/10.5755/j01.mech.22.6.16891 